PROCEEDINGS OF THE

AMERICAN MATHEMATICAL SOCIETY

Volume 127, Number 6, Pages 1805-1813

S 0002-9939(99)05015-7

Article electronically published on February 17, 1999

\title{
ON THE EIGENVALUE RATIO FOR VIBRATING STRINGS
}

\author{
MIN-JEI HUANG
}

(Communicated by Hal L. Smith)

\begin{abstract}
For vibrating strings with concave densities or symmetric singlebarrier densities, the ratio $\lambda_{2} / \lambda_{1}$ of the first two eigenvalues is minimized when the density is constant; while, for vibrating strings with symmetric single-well densities, the ratio $\lambda_{2} / \lambda_{1}$ is maximized when the density is constant.
\end{abstract}

\section{INTRODUCTION}

If a string with a nonnegative integrable density $\rho(x), x \in[0, a]$, is fixed at the end points $x=0$ and $x=a$ under unit tension, then the natural frequencies of vibration of the string are determined by the eigenvalues of the boundary value problem

$$
u^{\prime \prime}(x)+\lambda \rho(x) u(x)=0, \quad u(0)=u(a)=0 .
$$

As is well known, the eigenvalues of (1) form a strictly increasing sequence of positive numbers which depend on the density $\rho(x)$. We denote them accordingly by

$$
0<\lambda_{1}[\rho]<\lambda_{2}[\rho]<\lambda_{3}[\rho]<\cdots .
$$

Bounds on ratios of eigenvalues, especially the ratio of the first two eigenvalues, have long been of interest. Let $E(M, H)$ denote the class of integrable functions satisfying $\int_{0}^{a} \rho(x) d x=M$ and the inequalities $0 \leq \rho(x) \leq H, x \in[0, a]$. It was shown by Gentry and Banks [4] that

$$
\min _{\rho \in E(M, H)} \frac{\lambda_{2}[\rho]}{\lambda_{1}[\rho]}=\left[\frac{2 z_{1}(H / M)}{\pi}\right]^{2}
$$

where $z_{1}(t)$ is the least positive root of the equation $\tan z=z(1-t)$; and the unique density function $\rho_{0}$ at which this minimum is attained is defined by

$$
\rho_{0}(x)= \begin{cases}0, & M / 2 H<x<a-M / 2 H, \\ H, & \text { elsewhere }\end{cases}
$$

Keller [5] and Mahar and Willner [7] solved the problem of minimizing and maximizing $\lambda_{2}[\rho] / \lambda_{1}[\rho]$ over the class $C_{\alpha}$ of piecewise continuous functions satisfying the inequalities $0<\alpha \leq \rho(x) \leq 1, x \in[0, a]$. They found the extremizing density functions and the corresponding ratios. As in (2), the extremizing density in both

Received by the editors September 19, 1997.

1991 Mathematics Subject Classification. Primary 34L15.

Key words and phrases. Eigenvalue ratio, eigenfunction, concave density, symmetric single-well density. 
cases is symmetric about $x=a / 2$, has two jumps and takes only two values, $\alpha$ and 1. In a recent paper [3], Ashbaugh and Benguria considered the problem of finding bounds for eigenvalue ratios for general classes of Sturm-Liouville operators. For the vibrating string (1), assuming $\rho(x)$ is continuous with $0<\alpha \leq \rho(x) \leq 1$, $x \in[0, a]$, they established that

$$
\max \{1,4 \alpha\} \leq \frac{\lambda_{2}[\rho]}{\lambda_{1}[\rho]} \leq 1+\frac{3}{\alpha} .
$$

In this paper we are concerned with the ratio of the first two eigenvalues of (1) for certain classes of densities. It is assumed that the density functions are nonnegative and have a positive $L^{1}$-norm on $[0, a]$. By a symmetric single-well (resp. symmetric single-barrier) density on $[0, a]$ we mean a piecewise continuous function $\rho(x)$ on $[0, a]$ which is symmetric about $x=a / 2$ and monotone decreasing (resp. monotone increasing) on $[0, a / 2]$. We prove in $\S 3$ that if $\rho(x)$ is a symmetric single-well (resp. symmetric single-barrier) density, then

$$
\frac{\lambda_{2}[\rho]}{\lambda_{1}[\rho]} \leq 4 \quad(\text { resp. } \geq 4)
$$

with equality if and only if $\rho(x)$ is a constant a.e. Much of our work in this section is inspired by the work of Ashbaugh and Benguria [2] and Lavine [6] who worked on the eigenvalue gap for Schrödinger operators. We should point out that the minimizing functions found by Gentry and Banks in [4] and by Keller in [5] are symmetric single-well densities, while the maximizing function found by Mahar and Willner in [7] is a symmetric single-barrier density. Finally, in $\S 4$ we consider the class of concave densities. We prove that if $\rho(x)$ is a concave density, then

$$
\frac{\lambda_{2}[\rho]}{\lambda_{1}[\rho]} \geq 4
$$

with equality if and only if $\rho(x)$ is a constant. Our approach is based on some ideas of Lavine [6], where it was shown that for Schrödinger operators on an interval with convex potentials, the gap between the first two eigenvalues is minimized when the potential is a constant.

\section{Preliminaries}

Let $u_{n}(x)$ be the $n$th eigenfunction of (1) corresponding to $\lambda_{n}[\rho]$, normalized so that

$$
\int_{0}^{a} \rho(x) u_{n}^{2}(x) d x=1 .
$$

It is well known that $u_{n}(x)$ has exactly $(n-1)$ zeros in the open interval $(0, a)$. We may assume that $u_{1}(x)>0$ on $(0, a)$, and that $u_{2}(x)>0$ on $\left(0, x_{0}\right)$ and $u_{2}(x)<0$ on $\left(x_{0}, a\right)$, where $u_{2}\left(x_{0}\right)=0$. We first note that if $\rho(x)>0$ on $(0, a)$, then $u_{2}(x) / u_{1}(x)$ is strictly decreasing on $(0, a)$. To see this, let $w(x)=u_{1}(x) u_{2}^{\prime}(x)-u_{1}^{\prime}(x) u_{2}(x)$ so that $\left[u_{2}(x) / u_{1}(x)\right]^{\prime}=w(x) / u_{1}^{2}(x)$. By the differential equation, we have

$$
w^{\prime}(x)=\left(\lambda_{1}-\lambda_{2}\right) \rho(x) u_{1}(x) u_{2}(x)
$$

which is negative on $\left(0, x_{0}\right)$ and positive on $\left(x_{0}, a\right)$. Since $w(0)=w(a)=0$, this implies that $w(x)<0$ on $(0, a)$. So, $u_{2}(x) / u_{1}(x)$ is strictly decreasing on $(0, a)$. As a result, there are points $x_{ \pm}$with

$$
0 \leq x_{-}<x_{0}<x_{+} \leq a
$$


such that

$$
\left\{\begin{array}{l}
u_{2}^{2}(x)>u_{1}^{2}(x) \text { on }\left(0, x_{-}\right) \cup\left(x_{+}, a\right), \\
u_{2}^{2}(x)<u_{1}^{2}(x) \text { on }\left(x_{-}, x_{+}\right)
\end{array}\right.
$$

Since $u_{1}(x)$ and $u_{2}(x)$ are normalized, the set $\left(0, x_{-}\right) \cup\left(x_{+}, a\right)$ is not empty.

Next, suppose $\rho(\cdot, t)$ is a one-parameter family of piecewise continuous densities such that $\frac{\partial \rho}{\partial t}(x, t)$ exists. Let $\lambda_{n}(t)$ be the $n$th eigenvalue of $(1)$ with $\rho=\rho(x, t)$, and let $u_{n}(x, t)$ be the corresponding normalized eigenfunction. The formula

$$
\frac{d}{d t} \lambda_{n}(t)=-\lambda_{n}(t) \int_{0}^{a} \frac{\partial \rho}{\partial t}(x, t) u_{n}^{2}(x, t) d x
$$

was derived by Keller in [5]. In fact, differentiation of the equation

$$
u_{n}^{\prime \prime}(x, t)+\lambda_{n}(t) \rho(x, t) u_{n}(x, t)=0
$$

with respect to $t$ gives

$$
\dot{u}_{n}^{\prime \prime}(x, t)+\lambda_{n}(t) \rho(x, t) \dot{u}_{n}(x, t)=-\dot{\lambda}_{n}(t) \rho(x, t) u_{n}(x, t)-\lambda_{n}(t) \dot{\rho}(x, t) u_{n}(x, t) .
$$

We multiply this equation by $u_{n}(x, t)$ and integrate with respect to $x$ from 0 to $a$. This gives

$$
\begin{gathered}
\int_{0}^{a} \dot{u}_{n}^{\prime \prime}(x, t) u_{n}(x, t) d x-\int_{0}^{a} u_{n}^{\prime \prime}(x, t) \dot{u}_{n}(x, t) d x \\
=-\dot{\lambda}_{n}(t)-\lambda_{n}(t) \int_{0}^{a} \dot{\rho}(x, t) u_{n}^{2}(x, t) d x
\end{gathered}
$$

where we have used (6) and the normalization condition

$$
\int_{0}^{a} \rho(x, t) u_{n}^{2}(x, t) d x=1 .
$$

Integrating by parts twice and using the boundary condition $u_{n}(0, t)=u_{n}(a, t)=0$, we find that the left-hand side of (7) equals zero. This establishes (5). From (5), we obtain the formula

$$
\frac{d}{d t}\left[\frac{\lambda_{n}(t)}{\lambda_{m}(t)}\right]=\frac{\lambda_{n}(t)}{\lambda_{m}(t)} \int_{0}^{a} \frac{\partial \rho}{\partial t}(x, t)\left[u_{m}^{2}(x, t)-u_{n}^{2}(x, t)\right] d x \quad(n, m=1,2,3, \ldots)
$$

which is important for our analysis in this paper.

\section{SymmetriC SINGLE-WELL DENSITIES}

Theorem 3.1. If $\rho(x)$ is a symmetric single-well density on $[0, a]$, then

$$
\frac{\lambda_{2}[\rho]}{\lambda_{1}[\rho]} \leq 4
$$

The equality holds if and only if $\rho(x)$ is a constant a.e.

Proof. Consider the one-parameter family of densities $\rho(x, t)=t \rho(x)+(1-t) \varepsilon$, where $0<t<1$ and $\varepsilon$ is a positive constant. As in the previous section, we denote by $\lambda_{n}(t)$ the $n$th eigenvalue of $(1)$ with $\rho=\rho(x, t)$ and by $u_{n}(x, t)$ the corresponding normalized eigenfunction. For each $t$, since $\rho(x, t)$ is symmetric about $x=a / 2$, the 
same is true of $u_{1}^{2}(x, t)$ and $u_{2}^{2}(x, t)$ as functions of $x$. So there are points $x_{ \pm}(t)$ with

$$
0<x_{-}(t)<\frac{a}{2}<x_{+}(t)<a, x_{-}(t)+x_{+}(t)=a
$$

such that

$$
\left\{\begin{array}{l}
u_{2}^{2}(x, t)>u_{1}^{2}(x, t) \text { on }\left(0, x_{-}(t)\right) \cup\left(x_{+}(t), a\right), \\
u_{2}^{2}(x, t)<u_{1}^{2}(x, t) \text { on }\left(x_{-}(t), x_{+}(t)\right)
\end{array}\right.
$$

by (4). We now show that $\frac{d}{d t}\left[\frac{\lambda_{2}(t)}{\lambda_{1}(t)}\right] \leq 0$ for $0<t<1$. From (8), we have

$$
\frac{d}{d t}\left[\frac{\lambda_{2}(t)}{\lambda_{1}(t)}\right]=\frac{\lambda_{2}(t)}{\lambda_{1}(t)} \int_{0}^{a}[\rho(x)-\varepsilon]\left[u_{1}^{2}(x, t)-u_{2}^{2}(x, t)\right] d x .
$$

Since $\rho(x)$ is a symmetric single-well density, we have

$$
\begin{aligned}
\int_{0}^{a} \rho(x)\left[u_{1}^{2}(x, t)-u_{2}^{2}(x, t)\right] d x= & \int_{\left(0, x_{-}(t)\right) \cup\left(x_{+}(t), a\right)} \rho(x)\left[u_{1}^{2}(x, t)-u_{2}^{2}(x, t)\right] d x \\
& +\int_{x_{-}(t)}^{x_{+}(t)} \rho(x)\left[u_{1}^{2}(x, t)-u_{2}^{2}(x, t)\right] d x \\
\leq & \rho\left(x_{-}(t)\right) \int_{0}^{a}\left[u_{1}^{2}(x, t)-u_{2}^{2}(x, t)\right] d x
\end{aligned}
$$

on using (9) and (10). So,

$$
\int_{0}^{a}[\rho(x)-\varepsilon]\left[u_{1}^{2}(x, t)-u_{2}^{2}(x, t)\right] d x \leq\left[\rho\left(x_{-}(t)\right)-\varepsilon\right] \int_{0}^{a}\left[u_{1}^{2}(x, t)-u_{2}^{2}(x, t)\right] d x .
$$

The normalization condition $\int_{0}^{a}[t \rho(x)+(1-t) \varepsilon] u_{n}^{2}(x, t) d x=1$ gives

$$
\int_{0}^{a}\left[u_{1}^{2}(x, t)-u_{2}^{2}(x, t)\right] d x=\frac{t}{\varepsilon} \int_{0}^{a}[\rho(x)-\varepsilon]\left[u_{2}^{2}(x, t)-u_{1}^{2}(x, t)\right] d x .
$$

So, by (12), we obtain

$$
\left[\frac{\rho\left(x_{-}(t)\right) t}{\varepsilon}+(1-t)\right] \int_{0}^{a}[\rho(x)-\varepsilon]\left[u_{1}^{2}(x, t)-u_{2}^{2}(x, t)\right] d x \leq 0 .
$$

Since $0<t<1$, this implies that

$$
\int_{0}^{a}[\rho(x)-\varepsilon]\left[u_{1}^{2}(x, t)-u_{2}^{2}(x, t)\right] d x \leq 0
$$

from which it follows that $\frac{d}{d t}\left[\frac{\lambda_{2}(t)}{\lambda_{1}(t)}\right] \leq 0$. Thus, by the continuity of eigenvalues, we obtain

$$
\frac{\lambda_{2}[\rho]}{\lambda_{1}[\rho]}=\frac{\lambda_{2}(1)}{\lambda_{1}(1)} \leq \frac{\lambda_{2}(0)}{\lambda_{1}(0)}=\frac{\lambda_{2}[\varepsilon]}{\lambda_{1}[\varepsilon]}=4
$$

The equality occurs only if $\lambda_{2}(t) / \lambda_{1}(t)$ is a constant. In this case, the equality holds in (14), and we then have

$$
\int_{0}^{a} \rho(x)\left[u_{1}^{2}(x, t)-u_{2}^{2}(x, t)\right] d x=\int_{0}^{a}\left[u_{1}^{2}(x, t)-u_{2}^{2}(x, t)\right] d x=0
$$

by (13). This together with (11) implies that $\rho(x)$ is a constant a.e. 
There is a corresponding result to Theorem 3.1 for symmetric single-barrier densities which is given in the next theorem. The proof is similar to that of Theorem 3.1 .

Theorem 3.2. If $\rho(x)$ is a symmetric single-barrier density on $[0, a]$, then

$$
\frac{\lambda_{2}[\rho]}{\lambda_{1}[\rho]} \geq 4
$$

The equality holds if and only if $\rho(x)$ is a constant a.e.

It is interesting to give an alternative proof of Theorem 3.1 under the additional hypothesis that the density is differentiable. The argument here is similar to that in Ashbaugh-Benguria [1], where the ratio of the first two eigenvalues of Schrödinger operators with nonnegative potentials was discussed.

Let $u_{n}(x)$ be the $n$th normalized eigenfunction of (1) corresponding to $\lambda_{n}[\rho] \equiv$ $\lambda_{n}$. Set $f(x)=u_{1}^{2}(x)$ and $g(x)=\left[u_{1}^{\prime}(x) u_{2}(x)-u_{1}(x) u_{2}^{\prime}(x)\right] / u_{1}(x)$. A direct computation then gives that

$$
\begin{gathered}
f^{\prime \prime}=\left\{2\left(\frac{u_{1}^{\prime}}{u_{1}}\right)^{2}-2 \lambda_{1} \rho\right\} f \equiv w_{1} f \\
g^{\prime \prime}=\left\{\left(2 \lambda_{1}-\lambda_{2}\right) \rho+2\left(\frac{u_{1}^{\prime}}{u_{1}}\right)^{2}+\frac{\left(\lambda_{2}-\lambda_{1}\right) \rho^{\prime} u_{1} u_{2}}{u_{1}^{\prime} u_{2}-u_{1} u_{2}^{\prime}}\right\} g \equiv w_{2} g
\end{gathered}
$$

so that

$$
w_{2}-w_{1}=\left(4 \lambda_{1}-\lambda_{2}\right) \rho+\frac{\left(\lambda_{2}-\lambda_{1}\right) \rho^{\prime} u_{1} u_{2}}{u_{1}^{\prime} u_{2}-u_{1} u_{2}^{\prime}}
$$

We compute

$$
\begin{aligned}
\int_{0}^{a}\left(w_{2}-w_{1}\right) u_{1}^{4} d x & =\int_{0}^{a}\left(\frac{g^{\prime \prime}}{g}-\frac{f^{\prime \prime}}{f}\right) f^{2} d x=\int_{0}^{a} \frac{f}{g}\left(f g^{\prime}-f^{\prime} g\right)^{\prime} d x \\
& =\int_{0}^{a}\left(\frac{f g^{\prime}-f^{\prime} g}{g}\right)^{2} d x \geq 0
\end{aligned}
$$

where an integration by parts was used in the third step. It follows that

$$
\left(4 \lambda_{1}-\lambda_{2}\right) \int_{0}^{a} \rho(x) u_{1}^{4}(x) d x+\left(\lambda_{2}-\lambda_{1}\right) \int_{0}^{a} \frac{\rho^{\prime}(x) u_{1}^{5}(x) u_{2}(x)}{u_{1}^{\prime}(x) u_{2}(x)-u_{1}(x) u_{2}^{\prime}(x)} d x \geq 0 .
$$

Since $\rho(x)$ is a symmetric single-well density, we see that $\rho^{\prime}(x) u_{2}(x) \leq 0$ on $[0, a]$. Also, it was shown in $\S 2$ that $u_{1}^{\prime}(x) u_{2}(x)-u_{1}(x) u_{2}^{\prime}(x)>0$ on $(0, a)$. Thus, the second integral in (15) is nonpositive. From this, we obtain the bound $\lambda_{2}[\rho] / \lambda_{1}[\rho] \leq$ 4. The equality occurs only when $\rho^{\prime}(x) u_{2}(x) \equiv 0$, i.e., only when $\rho(x)$ is a constant.

Remark 3.3. Suppose that $\rho(x)$ is a continuous, symmetric single-well density on $[0, a]$. Consider $\rho(x, t)=t \rho(x)+(1-t) \varepsilon$, where $0<t<1$ and $\varepsilon=\frac{1}{a} \int_{0}^{a} \rho(x) d x>0$. Then by (5),

$$
\frac{d}{d t} \lambda_{1}(t)=-\lambda_{1}(t) \int_{0}^{a}[\rho(x)-\varepsilon] u_{1}^{2}(x, t) d x
$$


Using $\int_{0}^{a}[\rho(x)-\varepsilon] d x=0$, the hypothesis of $\rho(x)$, and the fact that $u_{1}(x, t)$ is symmetric about $x=a / 2$ and monotone increasing on $[0, a / 2]$, it is easy to show that

$$
\int_{0}^{a}[\rho(x)-\varepsilon] u_{1}^{2}(x, t) d x \leq 0 .
$$

Hence $\frac{d}{d t} \lambda_{1}(t) \geq 0$. This implies that

$$
\lambda_{1}[\rho] \geq \lambda_{1}[\varepsilon]=\frac{\pi^{2}}{a \int_{0}^{a} \rho(x) d x} .
$$

Also, if $\rho(x)$ is uniformly positive, we have from (3) and Theorem 3.1,

$$
\max \left\{1,4 \frac{\min \rho}{\max \rho}\right\} \leq \frac{\lambda_{2}[\rho]}{\lambda_{1}[\rho]} \leq 4 .
$$

This leads to a lower bound for $\lambda_{2}[\rho]$ :

$$
\lambda_{2}[\rho] \geq \frac{\pi^{2}}{a \int_{0}^{a} \rho(x) d x} \cdot \max \left\{1,4 \frac{\min \rho}{\max \rho}\right\} .
$$

On the other hand, we have the following bounds for the symmetric single-barrier density $\rho(x)$ :

$$
\begin{gathered}
\lambda_{1}[\rho] \leq \frac{\pi^{2}}{a \int_{0}^{a} \rho(x) d x}, \\
4 \leq \frac{\lambda_{2}[\rho]}{\lambda_{1}[\rho]} \leq 1+3 \frac{\max \rho}{\min \rho}, \\
\lambda_{2}[\rho] \leq \frac{\pi^{2}}{a \int_{0}^{a} \rho(x) d x} \cdot\left\{1+3 \frac{\max \rho}{\min \rho}\right\} .
\end{gathered}
$$

\section{Concave Densities}

We now turn to the consideration of (1) in the case where $\rho(x)$ is concave. Our main result is:

Theorem 4.1. If $\rho(x)$ is a concave density on $[0, a]$, then

$$
\frac{\lambda_{2}[\rho]}{\lambda_{1}[\rho]} \geq 4
$$

with equality if and only if $\rho(x)$ is a constant.

We will first consider the linear case and then handle the general case. We begin with a lemma.

Lemma 4.2. If $g$ is three times differentiable and $u$ satisfies

$$
u^{\prime \prime}(x)+\lambda \rho(x) u(x)=0, \quad 0 \leq x \leq a, \quad u(0)=u(a)=0,
$$

where $\rho$ is differentiable, then

$$
g(a) u^{\prime}(a)^{2}-g(0) u^{\prime}(0)^{2}=\int_{0}^{a}\left[2 \lambda g^{\prime}(x) \rho(x)+\lambda g(x) \rho^{\prime}(x)+\frac{1}{2} g^{\prime \prime \prime}(x)\right] u^{2}(x) d x .
$$


Proof. We have

$$
\begin{aligned}
g(a) & u^{\prime}(a)^{2}-g(0) u^{\prime}(0)^{2} \\
& =\int_{0}^{a} \frac{d}{d x}\left\{g(x)\left[u^{\prime}(x)^{2}+\lambda \rho(x) u^{2}(x)\right]\right\} d x \\
& =\int_{0}^{a}\left\{g^{\prime}(x)\left[u^{\prime}(x)^{2}+\lambda \rho(x) u^{2}(x)\right]+\lambda g(x) \rho^{\prime}(x) u^{2}(x)\right\} d x
\end{aligned}
$$

and

$$
\begin{aligned}
0 & =\int_{0}^{a} \frac{d}{d x}\left[g^{\prime}(x) u^{\prime}(x) u(x)\right] d x \\
& =\int_{0}^{a} g^{\prime}(x)\left[u^{\prime}(x)^{2}-\lambda \rho(x) u^{2}(x)\right] d x+\int_{0}^{a} g^{\prime \prime}(x) u^{\prime}(x) u(x) d x \\
& =\int_{0}^{a} g^{\prime}(x)\left[u^{\prime}(x)^{2}-\lambda \rho(x) u^{2}(x)\right] d x-\frac{1}{2} \int_{0}^{a} g^{\prime \prime \prime}(x) u^{2}(x) d x .
\end{aligned}
$$

Combining these gives the desired result.

Lemma 4.3. Consider the one-parameter family of linear densities $\rho(x, t)=t x+b$, where $t>0$ and $b$ is a positive constant. Let $\lambda_{n}(t)$ be the nth eigenvalue of (1) with $\rho=\rho(x, t)$. Then the ratio $\lambda_{2}(t) / \lambda_{1}(t)$ is a strictly increasing function of $t$.

Proof. By (8),

$$
\frac{d}{d t}\left[\frac{\lambda_{2}(t)}{\lambda_{1}(t)}\right]=\frac{\lambda_{2}(t)}{\lambda_{1}(t)} \int_{0}^{a} x\left[u_{1}^{2}(x, t)-u_{2}^{2}(x, t)\right] d x .
$$

So, it suffices to show that

$$
\langle x(t)\rangle \equiv \int_{0}^{a} x\left[u_{1}^{2}(x, t)-u_{2}^{2}(x, t)\right] d x>0 \quad \text { for all } t>0 .
$$

Taking $g(x)=x$ in Lemma 4.2, we get

$$
a u_{n}^{\prime}(a, t)^{2}=\lambda_{n}(t) \int_{0}^{a}(3 t x+2 b) u_{n}^{2}(x, t) d x,
$$

and, with $g(x)=x^{2}$,

$$
a^{2} u_{n}^{\prime}(a, t)^{2}=\lambda_{n}(t) \int_{0}^{a}\left(5 t x^{2}+4 b x\right) u_{n}^{2}(x, t) d x .
$$

So,

$$
5 t \int_{0}^{a} x^{2} u_{n}^{2}(x, t) d x=(3 a t-4 b) \int_{0}^{a} x u_{n}^{2}(x, t) d x+2 a b \int_{0}^{a} u_{n}^{2}(x, t) d x .
$$

The normalization condition makes

$$
\int_{0}^{a} u_{n}^{2}(x, t) d x=\frac{1}{b}-\frac{t}{b} \int_{0}^{a} x u_{n}^{2}(x, t) d x .
$$

So, by (17),

$$
\int_{0}^{a} x^{2} u_{n}^{2}(x, t) d x=\frac{2 a}{5 t}+\frac{a t-4 b}{5 t} \int_{0}^{a} x u_{n}^{2}(x, t) d x .
$$


It follows that

$$
\begin{gathered}
\int_{0}^{a}\left[u_{1}^{2}(x, t)-u_{2}^{2}(x, t)\right] d x=-\frac{t}{b}\langle x(t)\rangle, \\
\int_{0}^{a} x^{2}\left[u_{1}^{2}(x, t)-u_{2}^{2}(x, t)\right] d x=\frac{a t-4 b}{5 t}\langle x(t)\rangle .
\end{gathered}
$$

To prove (16), we first note that $\langle x(t)\rangle \neq 0$ for all $t>0$. For, if for some $t$, $\langle x(t)\rangle=0$, then by (18) and (19), we would have

$$
\int_{0}^{a}\left(A x^{2}+B x+C\right)\left[u_{1}^{2}(x, t)-u_{2}^{2}(x, t)\right] d x=0
$$

where $A, B$ and $C$ are arbitrary constants. This is impossible because there are points $x_{ \pm}(t)$ with $0 \leq x_{-}(t)<x_{0}(t)<x_{+}(t) \leq a$ such that

$$
\left\{\begin{array}{l}
u_{1}^{2}(x, t)-u_{2}^{2}(x, t)<0 \text { on }\left(0, x_{-}(t)\right) \cup\left(x_{+}(t), a\right), \\
u_{1}^{2}(x, t)-u_{2}^{2}(x, t)>0 \text { on }\left(x_{-}(t), x_{+}(t)\right)
\end{array}\right.
$$

by (4). Thus, $\langle x(t)\rangle \neq 0$ for all $t>0$, and so, by the continuity of $\langle x(t)\rangle$, either $\langle x(t)\rangle>0$ for all $t$ or $\langle x(t)\rangle<0$ for all $t$.

Now, suppose on the contrary that $\langle x(t)\rangle<0$ for all $t$. Then by (18) and (19), we see that for $t<4 b / a$,

$$
\int_{0}^{a}\left(A x^{2}+B x+C\right)\left[u_{1}^{2}(x, t)-u_{2}^{2}(x, t)\right] d x<0
$$

where $A<0, B>0$ and $C \leq 0$. But choosing

$$
A x^{2}+B x+C=-\left[x-x_{-}(t)\right]\left[x-x_{+}(t)\right]
$$

and using (20), we find that the integral in (21) is positive. This leads to a contradiction, and (16) is proved.

We also obtain from Lemma 4.3 the result that for $t>0$, the ratio

$$
\frac{\lambda_{2}[x+t]}{\lambda_{1}[x+t]}=\frac{\lambda_{2}\left[\frac{x}{t}+1\right]}{\lambda_{1}\left[\frac{x}{t}+1\right]}
$$

is a strictly decreasing function of $t$. So,

$$
\frac{\lambda_{2}[x]}{\lambda_{1}[x]}>\frac{\lambda_{2}[x+t]}{\lambda_{1}[x+t]}>4
$$

It is clear that if the density $\rho(x)$ in (1) is replaced by the density function $\bar{\rho}(x)=$ $\rho(a-x)$, then the eigenvalues of (1) remain unchanged. Thus, we have the following result:

Lemma 4.4. If the linear density $m x+b$ is positive on $(0, a)$, where $m \in \mathbf{R}$ and $b \geq 0$, then

$$
\frac{\lambda_{2}[m x+b]}{\lambda_{1}[m x+b]} \geq 4
$$

The equality holds if and only if $m=0$. 
We are now ready to prove Theorem 4.1.

Proof. By Lemma 4.4, we need only show that if $\rho(x)$ is concave but not linear, there is a linear density with a smaller ratio. Let $u_{n}(x)$ be the $n$th normalized eigenfunction corresponding to $\lambda_{n}[\rho]$. Then there are points $x_{ \pm}$such that (4) holds. Let $L_{\rho}(x)$ be the linear function satisfying $L_{\rho}\left(x_{ \pm}\right)=\rho\left(x_{ \pm}\right)$. Since $\rho(x)$ is concave and not linear, it follows that

$$
\int_{0}^{a}\left[L_{\rho}(x)-\rho(x)\right]\left[u_{1}^{2}(x)-u_{2}^{2}(x)\right] d x<0 .
$$

Now define a one-parameter family of densities:

$$
\left\{\begin{array}{l}
\rho(x, 0)=\rho(x) \\
\frac{\partial \rho}{\partial t}(x, t)=L_{\rho(\cdot, t)}(x)-\rho(x, t), \quad t \geq 0 .
\end{array}\right.
$$

Then, by (8) and (22),

$$
\frac{d}{d t}\left[\frac{\lambda_{2}(t)}{\lambda_{1}(t)}\right]=\frac{\lambda_{2}(t)}{\lambda_{1}(t)} \int_{0}^{a}\left[L_{\rho(\cdot, t)}(x)-\rho(x, t)\right]\left[u_{1}^{2}(x, t)-u_{2}^{2}(x, t)\right] d x<0 .
$$

So, $\lambda_{2}(t) / \lambda_{1}(t)$ is a strictly decreasing function of $t$. On the other hand, if

$$
L_{\rho(\cdot, t)}(x)=m(t) x+b(t),
$$

then

$$
\rho(x, t)=e^{-t} \rho(x)+\int_{0}^{t} e^{s-t}[m(s) x+b(s)] d s .
$$

Thus, $\rho(\cdot, t)$ differs from a linear function by at most $e^{-t}\|\rho\|_{\infty}$, and so, by the continuity of eigenvalues, the eigenvalue ratio $\lambda_{2}(t) / \lambda_{1}(t)$ of $\rho(\cdot, t)$ is arbitrarily close to that of the linear density if $t$ is sufficiently large. This proves the theorem.

\section{ACKNowledgments}

The author wishes to thank the referee for useful comments.

\section{REFERENCES}

[1] M. Ashbaugh and R. Benguria, On the ratio of the first two eigenvalues of Schrödinger operators with positive potentials, Differential Equations and Mathematical Physics (I. W. Knowles and Y. Saito, eds.), Lecture Notes in Math., vol. 1285, Springer-Verlag, Berlin, 1987, pp. 16-25. MR 89a:35151

[2] M. Ashbaugh and R. Benguria, Optimal lower bound for the gap between the first two eigenvalues of one-dimensional Schrödinger operators with symmetric single-well potentials, Proc. Amer. Math. Soc. 105 (1989), 419-424. MR 89f:81028

[3] M. Ashbaugh and R. Benguria, Eigenvalue ratios for Sturm-Liouville operators, J. Differential Equations 103 (1993), 205-219. MR 94c:34125

[4] R. D. Gentry and D. O. Banks, Bounds for functions of eigenvalues of vibrating systems, J. Math. Anal. Appl. 51 (1975), 100-128. MR 51:8528

[5] J. B. Keller, The minimum ratio of two eigenvalues, SIAM J. Appl. Math. 31 (1976), 485-491. MR 54:10737

[6] R. Lavine, The eigenvalue gap for one-dimensional convex potentials, Proc. Amer. Math. Soc. 121 (1994), 815-821. MR 94i:35144

[7] T. J. Mahar and B. E. Willner, An extremal eigenvalue problem, Comm. Pure Appl. Math. 29 (1976), 517-529. MR 54:13201

Department of Mathematics, National Tsing Hua University, Hsinchu, Taiwan 30043 E-mail address: mjhuang@math.nthu.edu.tw 